\title{
Pratiques alimentaires et productivité des femelles laitières en zone périurbaine de Dakar
}

\author{
M. Ba Diao ${ }^{1 *}$ A. Dieng ${ }^{2}$ M.M. Seck ${ }^{3}$ R.C. Ngomibé ${ }^{2}$
}

\begin{abstract}
Mots-clés
Bovin laitier - Alimentation des animaux - Production laitière Reproduction - Zone périurbaine Sénégal.
\end{abstract}

\begin{abstract}
Résumé
Les pratiques alimentaires et les performances de reproduction et de production laitière ont été étudiées dans une unité laitière dans la zone périurbaine de Dakar. Une enquête sur les pratiques alimentaires a été réalisée en septembre - octobre 2004. L'exploitation des données enregistrées a permis de calculer la production laitière moyenne par vache entre novembre 2003 et octobre 2004 et les paramètres de reproduction entre 1991 et 2004. La superficie fourragère (30 ha) et l'effectif de vaches présentes (84 vaches) étaient nettement plus élevés que ceux de la majorité des élevages laitiers périurbains de Dakar. Néanmoins, l'alimentation des animaux est restée fortement tributaire des apports de paille de riz et des concentrés. Les difficultés d'acquisition de ces intrants ont induit des changements fréquents de régimes alimentaires. En outre, comme il y avait un mélange de plusieurs types génétiques bovins très différents dans un même lot d'alimentation, les besoins quotidiens en éléments nutritifs de base ont été rarement couverts pour les fortes productrices. Ces mauvaises conditions d'élevage se sont traduites par une production laitière moyenne faible (1 $972 \mathrm{~kg} / \mathrm{vache} / \mathrm{an})$, un âge au premier vêlage élevé pour les génisses nées au Sénégal (37 mois) et des intervalles entre vêlages (17 mois) trop longs. L'analyse des facteurs influençant les résultats de reproduction a montré que l'intervalle entre vêlages était fortement lié aux effets du type génétique et des variations climatiques interannuelles. Ces observations indiquent l'importance des facteurs du milieu (climat, conduite alimentaire) sur les performances des vaches et la nécessité d'une gestion rigoureuse de l'alimentation en fonction du type génétique.
\end{abstract}

\section{INTRODUCTION}

Au Sénégal, l'Etat mène depuis plus de deux décennies une politique d'amélioration et d'intensification des productions animales, notamment de la production laitière. Assurer la sécurité alimentaire en lait et produits laitiers est une priorité pour les pouvoirs publics qui ont mis en œuvre une stratégie d'intensification basée sur l'exploitation de races bovines exotiques hautes productrices introduites en zone périurbaine des Niayes (1). Cependant, en dépit de nombreuses opérations d'appui technique et économique avec la mise en place du projet laitier et d'importants investissements de

\footnotetext{
1. Institut sénégalais de recherches agricoles, Laboratoire national de l'élevage et de recherches vétérinaires, BP 2057, Dakar, Sénégal.

Tél. : +2218323678/8327692; fax : +2218323679

E-mail : mbadiao@sentoo.sn

2. Ecole nationale supérieure d'agriculture, Thiès, Sénégal.

3. Université Cheikh Anta Diop, Dakar, Sénégal.

* Auteur pour la correspondance
}

la part des éleveurs (2), les prévisions de production pour la zone périurbaine de 15000 litres par jour (12) n’ont pas été atteintes. Le pays reste largement tributaire des importations qui ont coûté près de 37 milliards de francs CFA en 2004 (13).

Ce déficit de production laitière est notamment dû aux contraintes alimentaires du cheptel, accentuées par les insuffisances structurelles des exploitations, telles que le manque de superficie, le morcellement des exploitations, la faiblesse des compétences techniques des acteurs, et par la détérioration de l'environnement économique global de la production laitière $(2,7)$. Dans la zone périurbaine de Dakar, la majorité des fermes intensives sont de petites unités de production, d'une à dix vaches laitières, caractérisées par l'exiguïté de l'assise foncière ne leur permettant pas de cultiver du fourrage (2). En effet, la superficie moyenne réservée à l'élevage est de 2,6 ha et 47 p. 100 des fermes ont moins d'un hectare (21). L'utilisation d'une alimentation sèche à base de sous-produits agricoles et agro-industriels est donc généralisée chez les petits producteurs. Les performances obtenues sont faibles en raison, en grande partie, 
des difficultés liées à l'approvisionnement en intrants (2). Néanmoins, dans ce contexte, des entreprises à vocation laitière affirmée se sont constituées, avec comme objectif principal l'exploitation de vaches exotiques à haute potentialité génétique. La base de l'alimentation dans ces fermes spécialisées est le fourrage cultivé (maïs ou sorgho) vert ou ensilé. Cet article présente les pratiques alimentaires et les résultats techniques obtenus dans l'une de ces fermes laitières spécialisées installées en zone périurbaine de Dakar.

\section{MATERIEL ET METHODES}

\section{Présentation de l'unité laitière étudiée}

L'unité laitière étudiée était la ferme de Niacoulrab située à $30 \mathrm{~km}$ au nord-est de Dakar. Elle a été choisie sur la base de l'ancienneté et de la stabilité de l'activité de production laitière qui a démarré en 1991. Les activités horticoles (arboriculture fruitière et marâ̂chage) et celles d'élevage (bovins laitiers, bovins extensifs et ovins) y étaient associées sur une superficie totale de 85 ha. Les plantations d'agrumes, de manguiers, de papayers, de passiflores, de bananiers plantains et le maraîchage occupaient 61 p. 100 de la superficie. La surface fourragère (maïs) représentait 35 p. 100 du domaine.

L'atelier de production laitière était composé d'animaux de races importées et de métis demi ou trois quarts sang exotique (croisements entre Gobra et Holstein ou Montbéliarde), élevés en stabulation permanente. L'alimentation était à base d'ensilage de maïs et de paille de riz associé aux concentrés fabriqués à la ferme.

Les animaux de l'élevage extensif, de races locales, étaient conduits au pâturage de juillet à février - mars. Les femelles n'étaient pas traites et constituaient le troupeau de base pour la production de métis.

Les animaux bénéficiaient d'un suivi sanitaire permanent assuré par un vétérinaire qui était également responsable du suivi de la reproduction. Un technicien d'élevage était chargé de la production de fourrage, du rationnement, de l'abreuvement et de la traite mécanique.

Aucun contrôle individuel de la production laitière n'était effectué, faute d'installation d'un appareil de mesure adéquat au niveau de la traite mécanique. Les informations techniques disponibles concernaient les productions laitières mensuelles de l'ensemble du troupeau. Cependant, l'enregistrement des données individuelles de reproduction (vêlage, insémination, diagnostic de gestation) a été régulièrement mis à jour.

\section{Méthodes}

Seul l'atelier laitier a été concerné par la présente étude. Une enquête rétrospective sur les pratiques alimentaires sur un an a été réalisée en septembre - octobre 2004. Les quantités d'aliments distribuées aux différents lots d'animaux ont été notées. Elles ont été converties en unités fourragères lait (UFL) et en matières azotées digestibles (MAD) sur la base des tables de l'Inra (15) ou des analyses bromatologiques effectuées au Laboratoire national de l'élevage et de recherches vétérinaires de Dakar ou à l'Ecole nationale supérieure d'agriculture de Thiès. Une enquête rétrospective portant sur l'évolution sur un an des stocks alimentaires a permis d'identifier les contraintes pour un approvisionnement régulier en aliments de bétail.

L'exploitation des données enregistrées a permis de calculer, sur une année (de novembre 2003 à octobre 2004), la moyenne économique à l'échelle de l'exploitation : (production laitière totale/ somme des jours de présence) x 365 .
L'âge au premier vêlage et l'intervalle entre vêlages, critères d'appréciation des performances de reproduction, ont été calculés sur la base des enregistrements chronologiques des données entre 1991 et 2004. L'analyse statistique a été effectuée par calcul des moyennes et écarts-types. L'analyse de la variance a été utilisée pour comparer les moyennes. Sur l'âge au premier vêlage, les effets de l'origine (importée vs née au Sénégal), de la race (métisse, Montbéliarde, Holstein), du mois et de l'année de naissance ont été étudiés. Sur l'intervalle entre vêlages, les facteurs de variation introduits ont été la race, le rang, le mois et l'année de vêlage.

\section{RESULTATS}

\section{Race, effectifs et structure du troupeau}

Le recensement du troupeau laitier (tableau I) indique un effectif moyen de 218 animaux, dont 47,7 p. 100 de métis demi ou trois quarts sang exotique (croisés entre la race Gobra et la race Montbéliarde ou Holstein), 43,1 p. 100 de Holsteins, 7,0 p. 100 de Montbéliardes, 1,3 p. 100 de Jersiaise et 0,9 p. 100 d'Abondance. Les vaches, les jeunes femelles et les mâles ont représenté respectivement $38,5,34,0$ et 27,5 p. 100 de l'effectif. Parmi les vaches présentes, 27,4 p. 100 étaient improductives mais non réformées et un taux de vaches en lactation de 51,2 p. 100 a été observé.

\section{Tableau I}

Races et effectifs moyens dans l'unité de production étudiée

\begin{tabular}{lrcc} 
Race & Effectif moyen & \% Femelles & \% Vaches \\
\hline Métisse & 104 & 74 & 27 \\
Holstein & 94 & 74,5 & 51,1 \\
Montbéliarde & 15 & 40 & 26,7 \\
Abondance & 2 & 100 & 100 \\
Jersiaise & 3 & 100 & 66,7 \\
Total & 218 & 72,5 & 38,5
\end{tabular}

\section{Pratiques alimentaires}

Les animaux ont été répartis dans huit lots : vaches en production, vaches taries gravides, génisses premier âge (1-2 ans), génisses deuxième âge ( 2 ans - premier vêlage), femelles à la réforme non vendues, mâles, jeunes (6-12 mois) et veaux de moins de 6 mois. Le tableau II montre les poids vifs moyens et les rations alimentaires distribuées par lot. A l'intérieur de chaque lot, le régime alimentaire a été identique quels qu'aient été les races et les poids des animaux en présence.

Les veaux ont été séparés de leur mère dès la naissance. Ils ont été élevés dans la nursery où ils ont reçu quatre litres de lait par jour en deux repas jusqu'au sevrage intervenant à l'âge de 4 mois (races exotiques) ou de 5 à 6 mois (métis). Au-delà de deux semaines d'âge, $200 \mathrm{~g}$ d'un concentré de production [CP = 1,18 UFL, $219 \mathrm{~g}$ $\mathrm{MAD}, 1,1 \mathrm{~g}$ Ca et 4,8 $\mathrm{g} P$ par kilogramme de matière sèche (MS)] et de la paille de riz leur ont été offerts.

La ration de base a été la paille de riz non traitée, distribuée à l'ensemble des animaux âgés de plus de 6 mois et complétée par un concentré d'équilibre (CE = 0,96 UFL, 303 g MAD, 3,3 g Ca et 


\section{Tableau II}

Poids moyens des animaux et rations moyennes distribuées par lot d'alimentation dans l'unité de production étudiée

\begin{tabular}{|c|c|c|c|c|c|c|c|c|c|c|c|c|}
\hline \multirow{2}{*}{ Lot } & \multirow{2}{*}{ Caractéristiques } & \multirow{2}{*}{ Effectifs } & \multirow{2}{*}{$\begin{array}{l}\text { Poids vifs } \\
\text { moyens }\end{array}$} & \multicolumn{5}{|c|}{$\begin{array}{l}\text { Quantités d'aliments distribuées } \\
\text { (kg MS/animal/jour) }\end{array}$} & \multicolumn{4}{|c|}{$\begin{array}{l}\text { Valeurs alimentaires } \\
\text { (/animal/jour) }\end{array}$} \\
\hline & & & & Paille de riz & Ensilage & CE & CP & Total & UFL & MAD (g) & $\mathrm{Ca}(\mathrm{g})$ & $\mathbf{P}(\mathrm{g})$ \\
\hline 1 & Jeunes & & & & & & & & & & & \\
\hline & 6-12 mois & 18 & $119,2 \pm 16,9$ & 1,5 & - & 0,5 & 1,0 & 3,0 & 2,4 & 375 & 6,5 & 9,1 \\
\hline 2 & $\begin{array}{l}\text { Génisses } \\
\text { (12-24 mois) }\end{array}$ & 18 & $251,7 \pm 51,6$ & 3,5 & 1,1 & 1,3 & - & 5,9 & 4,0 & 446 & 16,3 & 12,8 \\
\hline 3 & $\begin{array}{l}\text { Génisses } \\
\text { ( } 24 \text { mois-1 } 1^{\text {er }} \text { vêlage) }\end{array}$ & 28 & $410,0 \pm 74,5$ & 5,7 & 0,9 & 0,8 & - & 7,4 & 4,4 & 294 & 19,6 & 12,2 \\
\hline 4 & Vaches en lactation & 43 & $577,1 \pm 100,0$ & 8,0 & - & 2,4 & 2,2 & 12,6 & 8,9 & 1232 & 29,8 & 31,6 \\
\hline 5 & Vaches taries & 18 & $517,8 \pm 115,2$ & 7,3 & - & 2,0 & 0,4 & 9,7 & 6,1 & 716 & 25,3 & 20,4 \\
\hline 6 & Femelles à la réforme & 24 & $486,8 \pm 83,4$ & 6,8 & - & 0,8 & - & 7,6 & 4,2 & 262,8 & 19,6 & 11,6 \\
\hline 7 & $\begin{array}{l}\text { Mâles âgés } \\
\text { de plus de } 12 \text { mois }\end{array}$ & 49 & $480,5 \pm 106,0$ & 6,7 & 0,5 & 0,5 & - & 7,7 & 4,3 & 191 & 19,9 & 11,0 \\
\hline
\end{tabular}

MS : matière sèche

CE : concentré d'équilibre $(60,7 \%$ drèche de brasserie ; $18,7 \%$ tourteau d'arachide ; $9,8 \%$ mélasse ; 9,3\% graine de coton ; $1,5 \%$ complément minéral vitaminé)

$\mathrm{CP}$ : concentré de production (61,5\% maïs grain ; $37 \%$ tourteau d'arachide ; $1,5 \%$ complément minéral vitaminé)

UFL : unités fourragères lait

MAD : matières azotées digestibles

5,2 g P / kg MS). De l'ensilage de maïs, mal conservé et moisi, a été offert aux génisses et aux mâles âgés de plus de 12 mois. Un concentré de production de même composition que celui des veaux a été donné aux femelles en lactation en priorité et parfois aux jeunes âgés de 6 à 12 mois et aux femelles taries.

La composition de ces concentrés a beaucoup varié en fonction de la disponibilité des intrants. Le tableau III montre l'évolution des stocks des différents intrants alimentaires de novembre 2003 à octobre 2004. Les ruptures de stocks de maïs et de tourteau d'arachide utilisés dans la fabrication de ces concentrés ont été fréquentes, conduisant à une variation de la composition des rations distribuées.

La quantité moyenne de MS consommée par vache en lactation et par jour a été de 12,6 kg. Les apports quotidiens moyens en éléments nutritifs de cette ration ont été de 8,9 UFL, 1232 g MAD, 29,8 g Ca, 31,6 g P. Les concentrés consommés ont représenté respectivement 36,5, 54,6 et 98,1 p. 100 des apports en MS, UFL et MAD.

\section{Performances de production laitière}

Sur l'ensemble du troupeau, le stade de lactation moyen a été de sept mois et 16 p. 100 des vaches ont dépassé 15 mois de lactation. La production annuelle moyenne (tableau IV) a été de $1972 \mathrm{~kg}$ de lait par vache présente, soit une production journalière moyenne de $5,5 \mathrm{~kg}$. Les femelles exotiques (dont 88 p. $100 \mathrm{de}$ Holsteins) ont produit $2523 \mathrm{~kg}$ par vache présente contre $869 \mathrm{~kg}$ pour les femelles métisses.

\section{Tableau III}

Quantités d'intrants alimentaires stockés (tonnes)

\begin{tabular}{|c|c|c|c|c|c|c|c|c|c|c|c|c|}
\hline \multirow{2}{*}{ Aliments } & \multicolumn{2}{|c|}{2003} & \multicolumn{10}{|c|}{2004} \\
\hline & Nov. & Déc. & Janv. & Fév. & Mars & Avr. & Mai & Juin & Juil. & Août & Sept. & Oct. \\
\hline Maïs & 0 & 9 & 4,5 & 9 & 0 & 4,5 & 0 & 4,5 & 4,5 & 4,5 & 4,2 & 0 \\
\hline Drêche & 77,2 & 91,3 & 70,2 & 63,2 & 57,6 & 35,1 & 28,1 & 63,2 & 77,2 & 0 & 14 & 14 \\
\hline Graine de coton & \multicolumn{12}{|c|}{$148 *$} \\
\hline Tourteau d'arachide & 5 & 5,5 & 5 & 0 & 10 & 0 & 4,14 & 1,3 & 5,1 & 5 & 5 & 0 \\
\hline Aliment commercial & 0 & 0 & 0 & 0 & 0 & 0 & 0 & 0 & 10 & 0 & 2 & 0 \\
\hline Mélasse & \multicolumn{12}{|c|}{$54 *$} \\
\hline
\end{tabular}

* Quantité stockée sur toute la période 
Tableau IV

Performances de production laitière dans l'unité de production étudiée

\begin{tabular}{lccc} 
& Effectifs & $\begin{array}{c}\text { Production } \\
\text { totale } \\
\text { (kg/an) }\end{array}$ & $\begin{array}{c}\text { Production } \\
\text { journalière } \\
\text { (kg/j) }\end{array}$ \\
\hline $\begin{array}{l}\text { Total vaches } \\
\text { présentes }\end{array}$ & 84 & 1972 & 5,5 \\
$\begin{array}{l}\text { Vaches exotiques } \\
(48 \text { HO, } 4 \text { MTB, 2 AB, 2 JER) }\end{array}$ & 56 & 2523 & 6,9 \\
$\begin{array}{l}\text { Vaches métisses } \\
(1 / 2 \text { et } 3 / 4 \text { de sang HO ou MTB) }\end{array}$ & 28 & 869 & 2,4 \\
\hline
\end{tabular}

HO : Holstein ; MTB : Montbéliarde ; JER : Jersiaise ; AB : Abondance

\section{Performances de reproduction}

Le tableau $\mathrm{V}$ montre les performances de reproduction obtenues. L'âge au premier vêlage a été de 34 mois avec un taux de variation de 23,4 p. 100. L'analyse de variance a montré que seul le facteur origine a eu une influence significative sur ce critère $(\mathrm{P}<0,001)$. Les génisses nées au Sénégal ont vêlé pour la première fois à 37 mois, c'est-à-dire 9 mois plus tard que les femelles importées (28 mois). La race n'a exercé aucun effet significatif sur l'âge au premier vêlage.

L'intervalle entre vêlages a été de 17 mois avec un taux de variation de 37,3 p. 100. Cette variation a été significativement influencée par la race et l'année de vêlage $(\mathrm{P}<0,001)$, alors que les effets de la saison et du rang de vêlage n'ont pas été significatifs dans le modèle utilisé. La race Holstein a donné les plus mauvais résultats ; l'intervalle a été rallongé de trois à quatre mois par rapport aux autres races présentes (tableau $\mathrm{V}$ ). La figure 1 montre que l'intervalle entre vêlages a varié selon les années.

\section{Tableau V}

Performances de reproduction dans l'unité de production étudiée

\section{Paramètres de reproduction}

Nb. d'animaux

Age $1^{\text {er }}$ vêlage

Toutes femelles Importée 287

125

Née au Sénégal

162

$025 \pm 240$

$832 \pm 41^{\mathrm{a}}$

$1110 \pm 33^{b}$

Intervalle entre vêlages

$\begin{array}{lrl}\text { Toutes femelles } & 440 & 518 \pm 193 \\ \text { Jersiaise } & 24 & 478 \pm 44^{\mathrm{a}} \\ \text { Montbéliarde } & 189 & 488 \pm 33^{\mathrm{a}} \\ \text { Métisse } & 65 & 506 \pm 44^{\mathrm{a}} \\ \text { Holstein } & 162 & 602 \pm 39^{\mathrm{b}}\end{array}$

a, b Dans le même bloc les moyennes affectées de lettres différentes sont significativement différentes $(\mathrm{P}<0,001)$

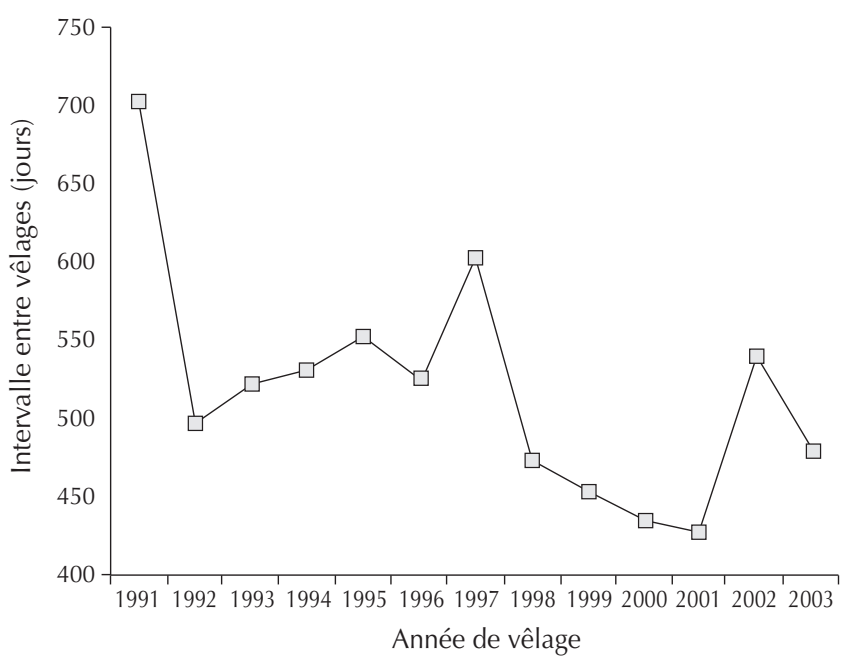

Figure 1 : influence de l'année de vêlage sur l'intervalle entre vêlages. Moyennes estimées par la méthode des moindres carrés.

\section{DISCUSSION}

Avec ses 30 hectares de surface fourragère et ses 84 vaches dont 51,2 p. 100 en production, la ferme de Niacoulrab est de taille relativement grande par rapport à la moyenne de la région des Niayes $(2,21)$. En effet, plus de 85 p. 100 des fermes laitières dans cette zone exploitaient moins de dix vaches et des superficies comprises entre un et cinq hectares. Néanmoins, dans la composition du troupeau laitier, la proportion de vaches présentes $(38,5$ p. 100) était faible par rapport à ce qui est habituel en élevage laitier spécialisé ; au Maroc, par exemple, la moyenne est de 58,5 p. 100 (23). Cette faible proportion de vaches s'explique par la présence d'un nombre important de mâles (29 p. 100). En outre, le maintien d'un lot de femelles improductives, qui représentaient 27,4 p. 100 des vaches, témoignait d'une mauvaise politique de réforme en vigueur dans cette ferme. Ces pratiques n'obéissent à aucune logique de recherche d'une productivité optimale.

Dans cette ferme, l'ensilage de maïs était normalement l'aliment de base pour les laitières. Avec la panne de l'ensileuse, un problème de conservation des fourrages s'est posé durant la campagne agricole 2003-2004. La paille de riz étant utilisée comme aliment de lest, il est certain que les concentrés ingérés par les vaches ont couvert aussi une bonne partie de leurs besoins d'entretien. Or, la forte variabilité dans la disponibilité des intrants entrant dans la composition de ces concentrés se traduit par un changement fréquent de régimes alimentaires. D'une part, ceci ne permet pas une accoutumance de la flore du rumen et, d'autre part, cela exige de la part du technicien d'élevage une grande maîtrise du rationnement.

La prévision correcte des quantités ingérées est une étape prépondérante du rationnement des vaches laitières (8). Pourtant dans cette ferme, les races n'étaient pas différenciées dans la gestion de l'alimentation. Or, la capacité d'ingestion d'une vache dépend du volume de son rumen, lui-même fonction de son poids et de son niveau de production. Elle est donc plus importante chez la Holstein que chez la Montbéliarde et la Jersiaise (20). On peut craindre alors un nivellement de la production vers le bas, un aplatissement de la courbe de lactation, surtout chez les fortes productrices en début de lactation, et un engraissement des vaches qui sont en fin de lactation ou qui sont moins bonnes laitières. Dans tous les cas, les régimes alimentaires offerts ont été très déséquilibrés et l'apport quotidien en énergie, en minéraux et parfois en matières azotées, très insuffisant pour une croissance régulière des génisses et une production laitière 
soutenue chez les hautes productrices. A titre d'exemple, le niveau des UF du régime offert aux vaches en production aurait permis de couvrir les besoins d'entretien et de production de 7 à $8 \mathrm{~kg}$ de lait pour une vache de $600 \mathrm{~kg}$. En revanche, les MAD autoriseraient trois à quatre fois plus de lait. Ces mauvaises conditions d'élevage se sont traduites par une moyenne économique faible (1 $972 \mathrm{~kg} / \mathrm{vache} / \mathrm{an})$, même pour les races exotiques $(2523 \mathrm{~kg} / \mathrm{vache} / \mathrm{an})$, comparée aux résultats disponibles dans certains pays tropicaux et subtropicaux où la Holstein-Frisonne produit entre 4000 et $6600 \mathrm{~kg}$ de lait par lactation (tableau VI). Les intervalles entre vêlages (17 mois) ont été également trop longs eu égard à l'optimum recherché (12-13 mois) et aux références bibliographiques disponibles (tableau VI). Parmi les races présentes, la Holstein a eu les plus mauvais résultats de reproduction, ce qui peut être expliqué par son potentiel génétique supérieur, donc par sa plus grande capacité à mobiliser ses réserves corporelles pour la production laitière en début de lactation (20). Or, si le poids de la femelle diminue entre le vêlage et la mise à la reproduction, la fécondation est difficile (14). L'année de vêlage influence également les performances de reproduction. En élevage bovin laitier dans des zones à aléas climatiques prononcés, les performances de reproduction sont liées au climat et à ses variations annuelles, de par leurs effets directs sur l'animal et indirects sur la production fourragère (5).

Les conditions d'alimentation des génisses, se traduisant par des déficits alimentaires chroniques, ne permettent pas un développement harmonieux et complet de l'organisme. Or, les caractéristiques de la croissance des génisses (vitesse et régularité de la croissance, poids au premier vêlage) ont une grande influence sur les performances ultérieures de la vache adulte : fertilité et surtout longévité de la femelle adulte (3). L'âge au premier vêlage observé chez les femelles nées au Sénégal (37 mois) traduit certainement un retard de maturité sexuelle. Ce résultat est comparable à ceux trouvés dans les petits élevages de la même zone des Niayes (2),

\section{Tableau VI}

Performances de reproduction et de production laitière dans divers pays

\begin{tabular}{|c|c|c|c|c|}
\hline Race & $\begin{array}{l}\text { Pays } \\
\text { (auteur) }\end{array}$ & $\begin{array}{l}\text { Age } 1^{\mathrm{er}} \text { vêlage } \\
\text { (mois) }\end{array}$ & $\begin{array}{l}\text { Intervalle entre vêlages } \\
\text { (jours) }\end{array}$ & $\begin{array}{c}\text { Production laitière } \\
\text { par lactation (kg) } \\
\text { (durée de lactation, en jours) }\end{array}$ \\
\hline Holstein & $\begin{array}{l}\text { Maroc - tous élevages } \\
\text { (Boujenane, 1986) }\end{array}$ & $29,5 \pm 3,5$ & $411 \pm 88$ & $3300(338)$ \\
\hline Holstein & $\begin{array}{l}\text { Guadeloupe - Martinique } \\
\text { (Jordan et coll., 1992) }\end{array}$ & $32,4 \pm 6$ & $446 \pm 123$ & $4367(330)$ \\
\hline Holstein - Frisonne & $\begin{array}{l}\text { Ghana } \\
\text { (Osei et coll., 1991) }\end{array}$ & $34,1 \pm 1,27$ & $480 \pm 67,5$ & $2499 \pm 148$ \\
\hline Holstein - Frisonne & $\begin{array}{l}\text { Kenya } \\
\text { (Staal et coll., 1998) }\end{array}$ & - & - & 4477 (305) \\
\hline Frisonnes (Pie-noir et Pie rouge) & $\begin{array}{l}\text { Maroc - fermes d'Etat } \\
\text { (Srairi et Kessab, 1998) }\end{array}$ & $30,2 \pm 1,8$ & $391 \pm 19$ & $\begin{array}{c}6016 \pm 836 \\
\text { (/vache/an) }\end{array}$ \\
\hline Holstein - Frisonne & $\begin{array}{l}\text { Cameroun } \\
\text { (Njwe et coll., 2002) }\end{array}$ & $32,5 \pm 3,5$ & - & $\begin{array}{c}4284 \pm 1626 \\
(315 \pm 36)\end{array}$ \\
\hline Jersiaise & $\begin{array}{l}\text { Sénégal } \\
\text { (Sow et Diop, 1996) }\end{array}$ & 30 & 428 & 3274 \\
\hline Montbéliarde & $\begin{array}{l}\text { Sénégal - station } \\
\text { (Denis, 1981) }\end{array}$ & 30 & $\begin{array}{r}484(\mathrm{I}) \\
390(\mathrm{~S})\end{array}$ & 3250 \\
\hline Diverses races laitières & $\begin{array}{l}\text { Sénégal - fermes périurbaines } \\
\text { (Ba Diao, 2005) }\end{array}$ & $\begin{array}{l}32 \pm 5(\mathrm{I}) \\
36 \pm 7(\mathrm{~S})\end{array}$ & $511 \pm 149$ & $\begin{array}{l}\text { Ho : } 4541 \pm 1730 \\
\text { Mt : } 3605 \pm 1356 \\
\text { Jer : } 3096 \pm 784\end{array}$ \\
\hline Zébu x Frison (F1) & $\begin{array}{l}\text { Ethiopie - Station Arsi } \\
\text { (Kiwuwa et coll., 1983) }\end{array}$ & - & 458 & $2352(373)$ \\
\hline $\begin{array}{l}\text { Ndama x Frisonne (F1) } \\
\text { Ndama x Jersey (F1) }\end{array}$ & $\begin{array}{l}\text { Gambie } \\
\text { (Diack et coll., 2004) }\end{array}$ & $\begin{array}{l}32,8 \pm 3,8 \\
31,9 \pm 2,5\end{array}$ & $\begin{array}{l}428,8 \pm 96,9 \\
382,7 \pm 67,2\end{array}$ & - \\
\hline Zebu x Frisonne (F1) & $\begin{array}{l}\text { Tanzanie } \\
\text { (Bee et coll., 2006) }\end{array}$ & - & $405 \pm 51$ & $2190 \pm 183$ \\
\hline $\begin{array}{l}\text { Boran x Frisonne (F1) } \\
\text { Frisonne } \\
\text { Boran x Jersey (F1) }\end{array}$ & $\begin{array}{l}\text { Ethiopie } \\
\text { (Demeke et coll., 2004) }\end{array}$ & $\begin{array}{r}36 \pm 0,4 \\
37,3 \pm 0,3 \\
35,4 \pm 0,5\end{array}$ & $\begin{array}{l}417 \pm 6 \\
459 \pm 4 \\
408 \pm 6\end{array}$ & - \\
\hline
\end{tabular}


et au Ghana (19). Ceux trouvés en Afrique de l'Est sont généralement meilleurs, entre 29 et 34 mois (tableau VI). Il existe donc des marges de progrès à réaliser durant la phase d'élevage des génisses dans les unités laitières des Niayes, afin de mieux maîtriser leur croissance et d'avancer l'âge au premier vêlage.

\section{CONCLUSION}

Malgré l'importance des superficies fourragères disponibles, cette unité laitière a affiché des résultats techniques (reproduction et production laitière) médiocres qui ne différaient guère de ceux obtenus au niveau des petits élevages laitiers périurbains. Ces résultats traduisent l'inadéquation des pratiques d'élevage, en particulier le non-respect des normes et recommandations alimentaires actuellement en vigueur en élevage laitier intensif, et le maintien d'animaux improductifs dans l'exploitation. La mise en œuvre d'une bonne politique de réforme devrait permettre d'améliorer l'offre alimentaire pour le reste du troupeau et d'augmenter sensiblement la productivité de l'élevage. L'analyse des performances de reproduction a montré que l'intervalle entre vêlages était lié à l'effet de la race ; ceci devrait amener les techniciens à mieux gérer le rationnement par type génétique. Une mauvaise conduite des génisses a été illustrée par leur âge moyen tardif au premier vêlage. De gros efforts devraient être davantage investis dans l'élevage des génisses, car en dépit de son caractère non immédiatement productif, il présente la clé de la réussite, surtout en raison de son incidence prononcée sur la carrière ultérieure des vaches laitières.

\section{Remerciements}

Les auteurs remercient l'ensemble du personnel technique de la ferme de Niacoulrab, et plus particulièrement K. Ba et M. Mboup pour leur disponibilité lors de la collecte des informations. Ils sont reconnaissants à $\mathrm{O}$. Bougaleb de l'Isra-Lnerv pour la relecture du document.

\section{BIBLIOGRAPHIE}

1. BA DIAO M., 1987. Un essai d'approche de l'encadrement en milieu intensif. Exemple du projet de développement de la production laitière intensive et semi-intensive dans la région des Niayes. Mémoire de confirmation, Isra-Lnerv, Dakar, Sénégal, 85 p.

2. BA DIAO M., 2005. Situation et conditions de développement de la production laitière intensive dans les Niayes au Sénégal. Thèse Doct. Biologie animale, Ucad, Dakar, Sénégal, 132 p.

3. BADINAND F., 1983. Relations fertilité - niveau de production alimentation. Inra Bull. tech. crzv Theix, 53 : 73-83.

4. BEE J.K.A., MSANGA Y.N., KAVANA P.Y., 2006. Lactation yield of crossbred dairy cattle under farmer management in Eastern coast of Tanzania. Livest. Res. rural Dev., 18: www.cipav.org.co/lrrd/lrrd18/2/ bee18023.htm.

5. BERBIGIER P., 1988. Bioclimatologie des ruminants domestiques en zone tropicale. Versailles, France, Inra, $237 \mathrm{p}$

6. BOUJENANE I., BA M., 1986. Performances de reproduction et de production laitière des vaches Pie noires au Maroc. Revue Elev. Méd. vét. Pays trop., 39 : 145-149.

7. BROUTIN C., SOKONA K., TANDIA A., BA M., 2000. Paysage des entreprises et environnement de la filière lait au Sénégal. Dakar, Sénégal, Gret / Enda-Graf / Ifan, 60 p. www.gret.org/incompe.

8. COULON J.B., HAUWUY A., MARTIN B., CHAMBA J.F., 1997. Pratiques d'élevage, production laitière et caractéristiques des fromages dans les Alpes du Nord. Prod. Anim., 10 : 195-205.

9. DEMEKE S., NESER F.W.C., SCHOEMAN S.J., 2004. Estimates of genetic parameters for Boran, Friesian and crosses of Friesian and Jersey with the Boran cattle in the tropical highlands of Ethiopia: reproduction traits. J. Anim. Breed. Genet., 121: 57-65.

10. DENIS J.P., 1981. Rapport sur la production laitière au Sénégal résultats des recherches entreprises durant le Ve plan. Dakar, Sénégal, Isra-Lnerv, $15 \mathrm{p}$

11. DIACK A., SANYANG F.B., CORR N., 2004. Survival, growth and reproductive performance in $\mathrm{F} 1$ crossbred cattle produced and managed on station in the Gambia. Livest. Res. rural Dev., 16: www.cipav.org.co/ Irrd//rrd16/9/diac16070.htm.

12. DiRECTION DE L'ELEVAGE, 1988. Plan d'action pour l'élevage. Dakar, Sénégal, ministère des Ressources animales, 76 p.

13. DIRECTION DE L'ELEVAGE, 2005. Productions animales en 2004. Dakar, Sénégal, direction de l'Elevage, 17 p.
14. GIROU R., 1969. La reproduction en fonction de I'alimentation. In: Alimentation des vaches laitières. Paris, France, Iteb / Inra, p. 133-136.

15. JARRIGE R., éd., 1988. Alimentation des bovins, ovins et caprins. Paris, France, Inra, $471 \mathrm{p}$.

16. JORDAN A., 1992. Situation et condition de développement du secteur productif au sein $d^{\prime}$ 'une filière laitière en milieu tropical insulaire : le cas des Antilles françaises. Thèse Doct., INA - Paris Grignon, France, 192 p. + annexes.

17. KIWUWA G.H., TRAIL I.C.M., KURTU M.Y., WORKU G., ANDERSON F.M., DURKIN J., 1983. Crossbred dairy cattle in Arsi Region, Ethiopia. Addis Ababa, Ethiopia, ILCA, p. 30. (Research report No. 11)

18. NJWE R.M., KWINJI L.N., GABCHE A.L., TAMBI E.N., 2002. Contributions of Heifers Project International (HPI) to small-scale dairy development in Cameroon. In: Rangnekar D., Thorpe W., Eds, Proc. South-South workshop Smallholder dairy production and marketing, opportunities and constraints, Anand, India, 13-16 March 2001. Anand, India, NDDB, Nairobi, Kenya, ILRI, p. 414-430.

19. OSEI S.A., EFFAH-BAAH K., KARIKARI P., 1991. The reproductive performance of Friesian cattle bred in the hot humid forest zone of Ghana. Anim. Genet. Res., 68: 490-497.

20. PRESTON T.R., 1988. Développement des systèmes de production laitière sous les Tropiques. Wageningen, Pays Bas, CTA, 71 p.

21. SERY A., 2003. Typologie des fermes laitières périurbaines de Dakar et Thiès. Thèse Doct. vét., Eismv, Dakar, Sénégal, 102 p.

22. SOW M.A., DIOP P.E.H., 1996. Place du système d'élevage intensif dans la production du lait au Sénégal ; exemple de la Société alimentaire (Soca). In : Diop P.E.H., Mazouz A., éds, Reproduction et production laitière. Paris, France, Aupelf-Uref, p. 75-80. (Coll. Universités francophones)

23. SRAIRI M.T., KESSAB B., 1998. Performances et modalités de production laitière dans six étables spécialisées au Maroc. Prod. Anim., $11: 321-326$.

24. STAAL S., CHEGE L., KENYANJUI M., KIMARI A., LUKUYU B., NJUBI D., OWANGO M., TANNER J., THORPE W., WAMBUGU M., 1998. Characterization of dairy systems supplying the Nairobi milk market: A pilot survey in Kiambu District for the identification of target groups of producers. Smallholder Dairy (R\&D) Project. Nairobi, Kenya, ILRI, p. 85.

Reçu le 02.11 .2005 , accepté le 10.08.2006 


\section{Summary}

Ba Diao M., Dieng A., Seck M.M., Ngomibé R.C. Feeding Practices and Productivity of Dairy Cows in the Suburban Area of Dakar

Feeding practices, and reproduction and milk production performances were studied in a dairy farm in the suburban area of Dakar. A survey on feeding practices was carried out in September-October 2004. The analysis of recorded data enabled to calculate the average milk production per cow between November 2003 and October 2004, and reproduction parameters between 1991 and 2004. The fodder area (30 ha) and the number of cows (84) were markedly higher than those of the majority of suburban Dakar dairy farms. Nevertheless, the animal feed depended still heavily on the supplies of rice straw and concentrates. The difficulties to acquire these inputs led to many changes in the feeding diets. Moreover, as there was a mixture of several and very different cattle genotypes within the same feeding groups, the daily requirements in basic nutritive elements were seldom met for high producing cows. These bad management conditions resulted in poor average milk production (1972 kg/cow/year), a late age at first calving for heifers born in Senegal (37 months) and long calving intervals (17 months). The analysis of the factors affecting the reproduction results showed that the calving interval was highly related to the genetic type and to interannual climatic variations. These observations show the importance of environmental factors (climate, feeding management) on cows' performances and the need to strictly adapt feeding practices to the genetic type.

Keywords: Dairy cattle - Animal feeding - Milk production Reproduction - Suburban area - Senegal.

\section{Resumen}

Ba Diao M., Dieng A., Seck M.M., Ngomibé R.C. Prácticas alimentarias y productividad de las hembras lecheras en las zonas periurbanas de Dakar

Las prácticas alimentarias y los rendimientos de reproducción y producción lechera han sido estudiadas en una unidad lechera de la zona periurbana de Dakar. Una encuesta sobre las prácticas alimentarias ha sido realizada en setiembre octubre 2004. La utilización de datos registrados ha permitido calcular el promedio de la producción lechera por vaca, entre noviembre 2003 y octubre 2004 y los parámetros de reproducción entre 1991 y 2004. La superficie de forraje (30 ha) y el efectivo de vacas presentes (84) fue apenas más elevado que el de la mayoría del ganado lechero periurbano de Dakar. Sin embargo, la alimentación de los animales se mantuvo fuertemente dependiente de los abastecimientos de paja de arrroz y de concentrados. La dificultad en la adquisición de éstos productos ha conducido a cambios frecuentes de regímenes alimentarios. Por otra parte, como había una mezcla de varios tipos genéticos de bovinos muy diferentes en un mismo lote de alimentación, las necesidades cotidianas en elementos nutritivos de bas fueron rara vez cubiertos para las productoras fuertes. Estas malas condiciones de cría se traducen en una producción lechera promedio baja (1972 kg/vaca/año), una edad elevada para el primer parto de los terneros nacidos en Senegal (37 meses) y muy largos los intervalos entre pateos (17 meses). El análisis de factores que afectan los resultados de reproducción ha mostrado que el intervalo entre parto está fuertemente ligado a los efectos de tipo genético y los cambios climáticos intra anuales. Esas observaciones indican la importancia de los factores ambientales (clima, conducta alimenticia) sobre el rendimiento de las vacas y la necesidad de una gestión rigurosa de la alimentación en función al tipo genético.

Palabras clave: Ganado bovino de leche - Alimentación de los animales - Producción lechera - Reproducción - Zona periurbana - Senegal. 